\title{
Intestinal Perforation as a Paradoxical Reaction to Antitubercular Therapy: A Case Report
}

\author{
Sung Hoon Kang ${ }^{1}$, Hee Seok Moon ${ }^{1}$, Jae Ho Park ${ }^{1}$, Ju Seok Kim ${ }^{1}$, Sun Hyung Kang ${ }^{1}$, Eaum Seok Lee ${ }^{1}$, \\ Seok Hyun Kim ${ }^{1}$, Byung Seok Lee ${ }^{1}$, Jae Kyu Sung ${ }^{1}$, Hyun Yong Jeong ${ }^{1}$, Kyung Ha Lee ${ }^{2}$ \\ ${ }^{1}$ Division of Gastroenterology, Department of Internal Medicine; ${ }^{2}$ Department of Surgery, Chungnam National University College of \\ Medicine, Daejeon, Korea
}

Paradoxical reactions to tuberculosis (TB) treatment are characterized by an initial improvement of the clinical symptoms followed by clinical or radiological deterioration of existing tuberculous lesions, or by development of new lesions. Intestinal perforation in gastrointestinal TB can occur as a paradoxical reaction to antitubercular therapy. A 55-year-old man visited the outpatient department with lower abdominal pain and weight loss. He was diagnosed with intestinal TB and started antitubercular therapy. After 3 months of antitubercular therapy, a colonoscopy revealed improvement of the disease. Three days after the colonoscopy, the patient visited the emergency room complaining of abdominal pain. Abdominal computed tomography revealed extraluminal air-filled spaces in the pelvic cavity. We diagnosed a small bowel perforation and performed an emergency laparotomy and a right hemicolectomy with small bowel resection. This report describes the case of intestinal perforation presenting as a paradoxical reaction to antitubercular and provides a brief literature review.

Keywords: Intestinal perforation; Extrapulmonary tuberculosis; Paradoxical reaction; Small intestine; Antitubercular therapy

\section{INTRODUCTION}

In 2018, extrapulmonary tuberculosis (TB) accounted for approximately $15 \%$ of all TB cases. Gastrointestinal TB accounts for 3\% to $19 \%$ of cases of extrapulmonary TB [1]. Complications of gastrointestinal TB include obstruction, fistula formation, and intestinal perforation [2]. The incidence of intestinal perforation due to gastrointestinal TB is $4 \%$ to $7.6 \%$, while the associated mortality rate is $30 \%$. While intestinal perforation can occur before or during antitubercular therapy [3], the latter presentation is rare and is suspected to be a possible paradoxical reaction.

Received: Feb 10, 2020 - Revised: Mar 13, 2020 - Accepted: Mar 16, 2020 Correspondence to: Hee Seok Moon

Division of Gastroenterology, Department of Internal Medicine, Chungnam National University College of Medicine, 282 Munhwa-ro, Jung-gu, Daejeon 35015 , Korea

Tel: +82-42-280-7036, Fax: +82-42-257-5753

E-mail:mhs1357@cnuh.co.kr

ORCID: https://orcid.org/0000-0002-8806-2163

(C) 2021 The Korean Society of Coloproctology

This is an open-access article distributed under the terms of the Creative Commons Attribution NonCommercial License (https://creativecommons.org/licenses/by-nc/4.0) which permits unrestricted noncommercial use, distribution, and reproduction in any medium, provided the original work is properly cited.
Paradoxical reactions to TB treatment are characterized by an initial improvement of clinical symptoms followed by clinical or radiological deterioration of existing tuberculous lesions or by the development of new lesions $[4,5]$. Paradoxical reaction is identified in $6 \%$ to $30 \%$ of patients receiving antitubercular therapy [5]. We report a case of intestinal perforation that occurred while a patient with normal immunity was being treated with antitubercular drugs for intestinal TB. This report was approved by the Institutional Review Board of Chungnam National University Hospital (CNUH 2020-01-017) and the written informed consent was received from the patient.

\section{CASE REPORT}

A 55-year-old man presented with a 2-month history of lower abdominal pain and diarrhea. He had lost $6 \mathrm{~kg}$ of weight since the symptoms started. Colonoscopy revealed transverse ulcerative lesions with clear borders and dirty exudates around the ileocecal valve (Fig. 1A). Histopathological examination revealed chronic granulomatous inflammation of the ileocecal mucosa with infiltration by histocytes and thin epithelioid cells (Fig. 1B). The TB polymerase chain reaction test was negative, but the interferon 

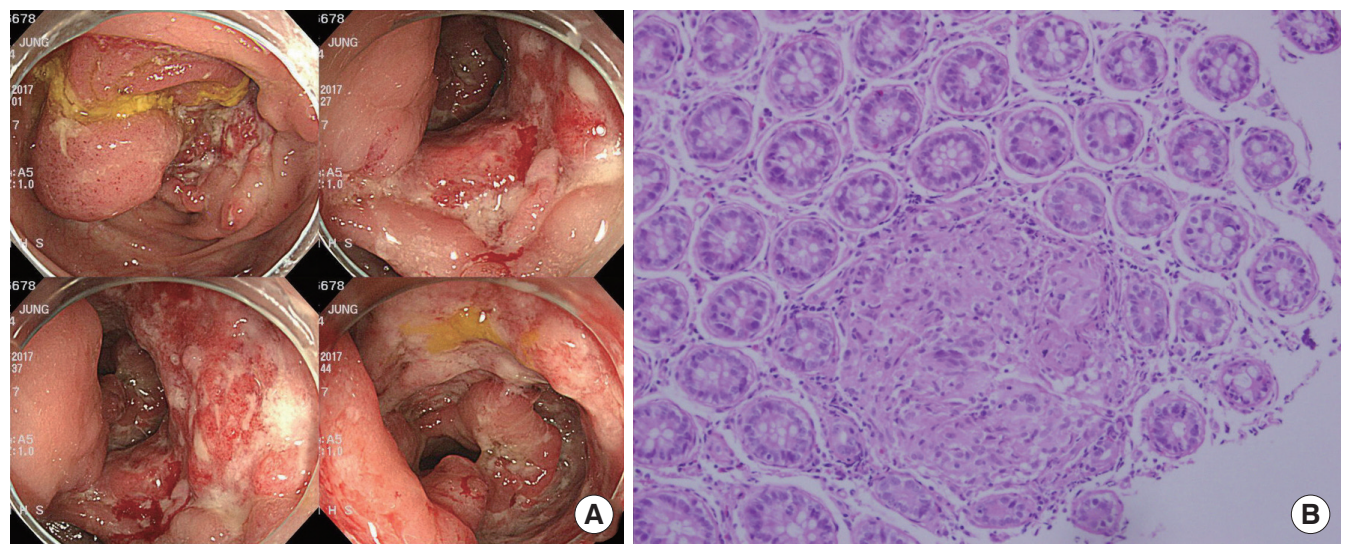

Fig. 1. (A) Photograph taken during the initial colonoscopy image showing transverse ulcerative lesions with clear borders and dirty exudates around the ileocecal valve. (B) Stained section of the ileocecal mucosa showing chronic granulomatous inflammation with infiltration by histocytes and thin epithelioid cells $(\mathrm{H} \& \mathrm{E}, \times 200)$.
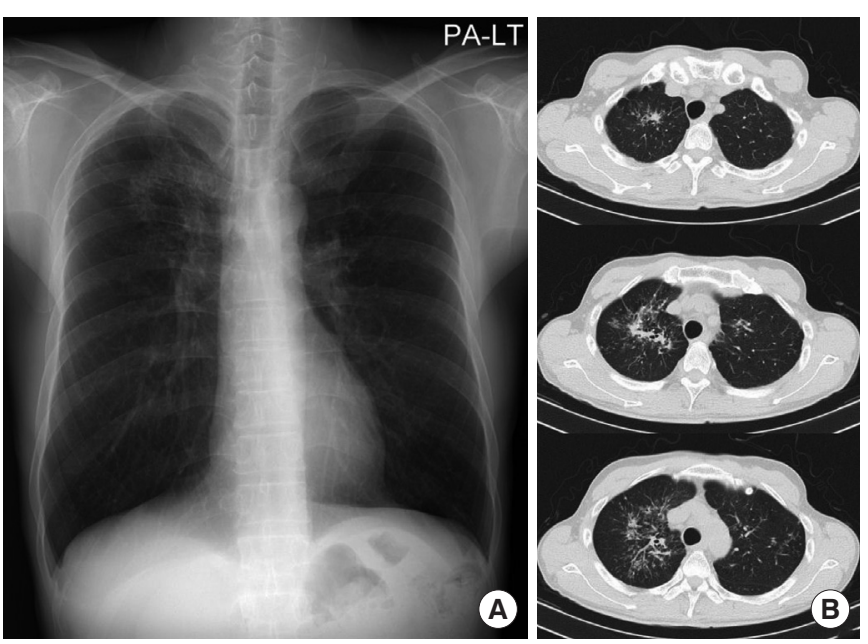

Fig. 2. (A) An image of the initial chest X-ray showing increased opacity of the right upper lobe. (B) Chest computed tomography, scan performed 4 weeks after starting antitubercular therapy, showing findings typical of active pulmonary tuberculosis, including infiltration and ill-defined centrilobular nodules with patchy opacity and bilateral multifocal tree-in-bud patterns in the upper lobes.

gamma release assay was positive, which helped us to determine the diagnosis of intestinal TB. The patient's chest X-ray showed increased opacity of the right upper lobe (Fig. 2A), but he had no respiratory symptoms, such as cough or expectoration. He began antitubercular therapy with a once-daily regimen of isoniazid (400 $\mathrm{mg}$ ), rifampin (600 mg), ethambutol (800 mg), and pyrazinamide (1,500 mg).

After 4 weeks of antitubercular therapy, the patient presented with cough and expectoration. The results of both sputum smear acid-fast bacilli (AFB) examination and sputum culture were negative. Chest computed tomography (CT) showed infiltration and ill-defined centrilobular nodules with patchy opacity and multifo- cal tree-in-bud patterns at bilateral upper lobes, leading to the diagnosis of active pulmonary TB (Fig. 2B).

After 2 months of undergoing the 4-drug therapy, the regimen was changed to a 3-drug regimen of isoniazid (400 mg), ethambutol (800 mg), and rifampin (600 mg) daily. The patient's symptoms of lower abdominal pain, diarrhea, cough, and expectoration were resolved, and he experienced no adverse effects apart from a mild rash.

A repeat colonoscopy was performed to evaluate treatment response after the patient completed 3 months of TB treatment. The ulcers, previously extending from the ileocecal valve till the cecum, had decreased in size. However, scars remained, associated with circular narrowing of the intestinal lumen. Localized inflammation had subsided (Fig. 3A). A microscopic examination of the ileocecal biopsy sample revealed chronic, nonspecific inflammation (Fig. 3B). We, therefore, decided to continue the patient on antitubercular drugs for a further 3 months as recommended.

Three days after the second colonoscopy, the patient came to the emergency room complaining of lower abdominal pain and constipation. On examination of his vital signs, his blood pressure, pulse rate, respiratory rate, and body temperature were $114 / 62$ $\mathrm{mmHg}, 86$ beats $/ \mathrm{min}, 24$ breaths $/ \mathrm{min}$, and $37.3^{\circ} \mathrm{C}$, respectively. Signs of localized tenderness and rebound tenderness were elicited on lower abdominal palpation. Laboratory tests showed a white blood cell (WBC) count of $14,800 / \mathrm{mm}^{3}$ (with $90.3 \%$ segmented neutrophils), hemoglobin of $15.4 \mathrm{~g} / \mathrm{dL}$, platelet count of $321,000 / \mathrm{mm}^{3}$, and a C-reactive protein (CRP) of $0.7 \mathrm{mg} / \mathrm{dL}$. Abdominal CT showed multisegmental wall thickening and luminal narrowing of the distal and terminal ileum, associated with partial small bowel obstruction, peritoneal inflammation, and complicated ascites (Fig. 4A). The patient was diagnosed with a small bowel obstruction with peritonitis. We performed bowel decompression using a Levin tube and administered antibiotics.

The patient did not report a lessening of lower abdominal pain, 


\section{$\begin{array}{rlr}\text { Coloproctology } & \text { Intestinal Perforation as a } & \text { Sung Hoon Kang, et al. }\end{array}$}
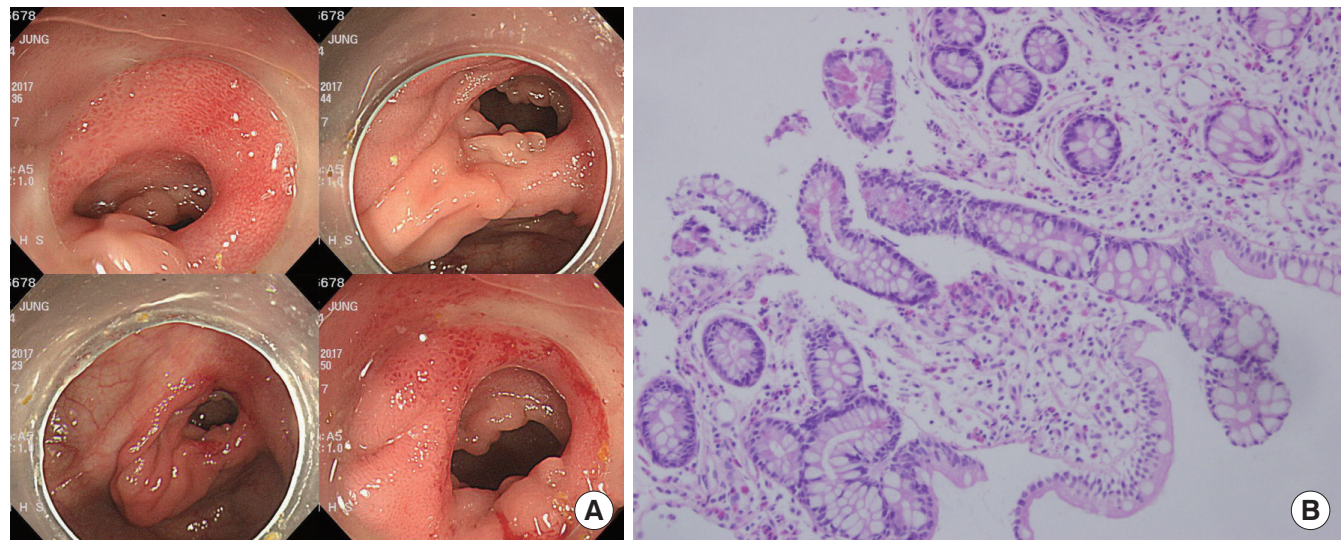

Fig. 3. (A) Colonoscopy image after completion of 12 weeks of antitubercular therapy showing reduced mucosal ulceration (which had previously extended from the ileocecal valve to the cecum), and an absence of inflammation, although scars with circular narrowing persist. (B) Histopathological examination of the intestinal biopsy sample (after completing 12 weeks of antitubercular therapy) showing chronic, nonspecific inflammation $(\mathrm{H} \& \mathrm{E}, \times 200)$.
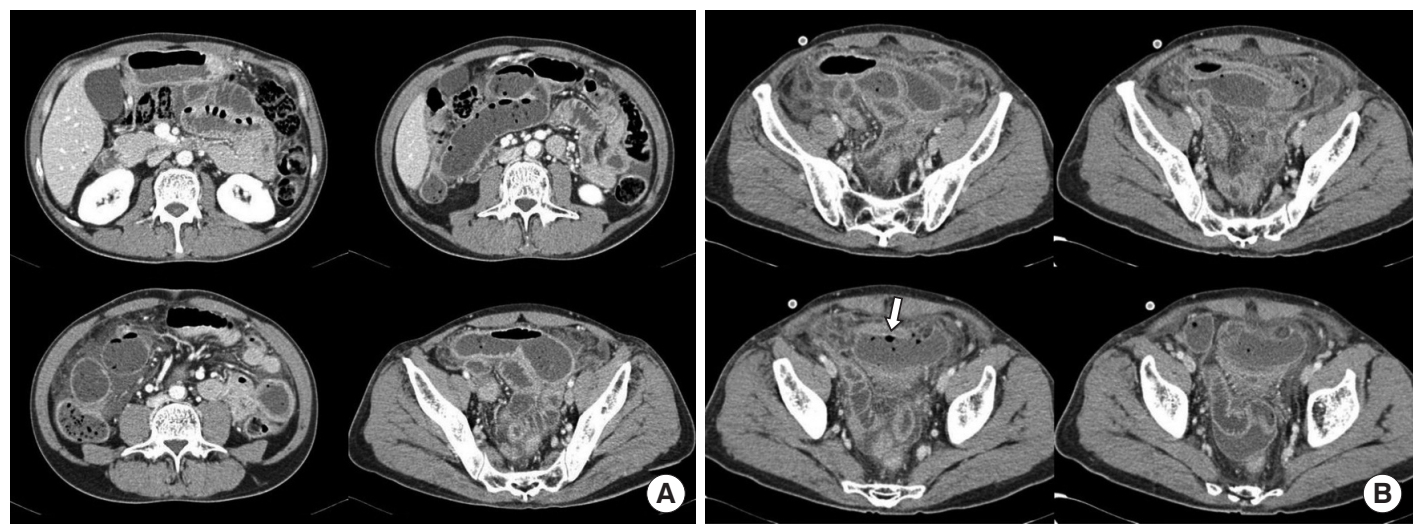

Fig. 4. (A) Abdominal computed tomography (CT) scan showing multisegmental wall thickening and luminal narrowing of the terminal and distal ileum with partial small bowel obstruction, peritonitis, and complicated ascites. (B) Repeat abdominal CT scan showing multiple, extraluminal air-filled spaces in the pelvic cavity, indicating intestinal perforation (arrow).

even on the second day of hospitalization, and developed a fever of $>38^{\circ} \mathrm{C}$. Blood tests revealed that his WBC count and CRP level had increased to $20,200 / \mathrm{mm}^{3}$ and $31.3 \mathrm{mg} / \mathrm{dL}$, respectively. A repeat abdominal CT revealed multiple, extraluminal, air-filled spaces in the pelvic cavity (Fig. 4B). We diagnosed small bowel perforation and performed an emergency laparotomy. Intraoperative examination revealed an ileal perforation at a distance of 100 $\mathrm{cm}$, proximal to the ileocecal valve. Some stricturing lesions were observed from the perforation site to the ileocecal valve.

We performed an ileocecectomy (Fig. 5A); 100-cm-long distal ileum and an 11-cm-long colon, along with the IC valve, were excised. Postoperative histopathological examination of the resected bowel revealed a perforated ulcer with acute transmural suppurative inflammation (Fig. 5B).

Five days after the operation, the patient resumed oral feeding and was discharged on the 8 th day after surgery. Antitubercular therapy was terminated after a further 3 months. The patient has been followed up for 2 years after the surgery and has remained asymptomatic. He has provided informed consent for the use of his medical information in this case report.

\section{DISCUSSION}

Extrapulmonary TB can affect lymph nodes, pleura, bones, the central nervous system, and the abdomen [2]. Gastrointestinal TB is the commonest presentation of abdominal TB and has been reported to account for $17 \%$ of all extrapulmonary cases.

Complications associated with gastrointestinal TB may include obstruction, perforation, fistula formation, and gastrointestinal bleeding. Risk factors for increased morbidity and mortality in patients with tuberculous intestinal perforation include delayed surgical treatment, multiple sites of perforation, concomitant cor- 

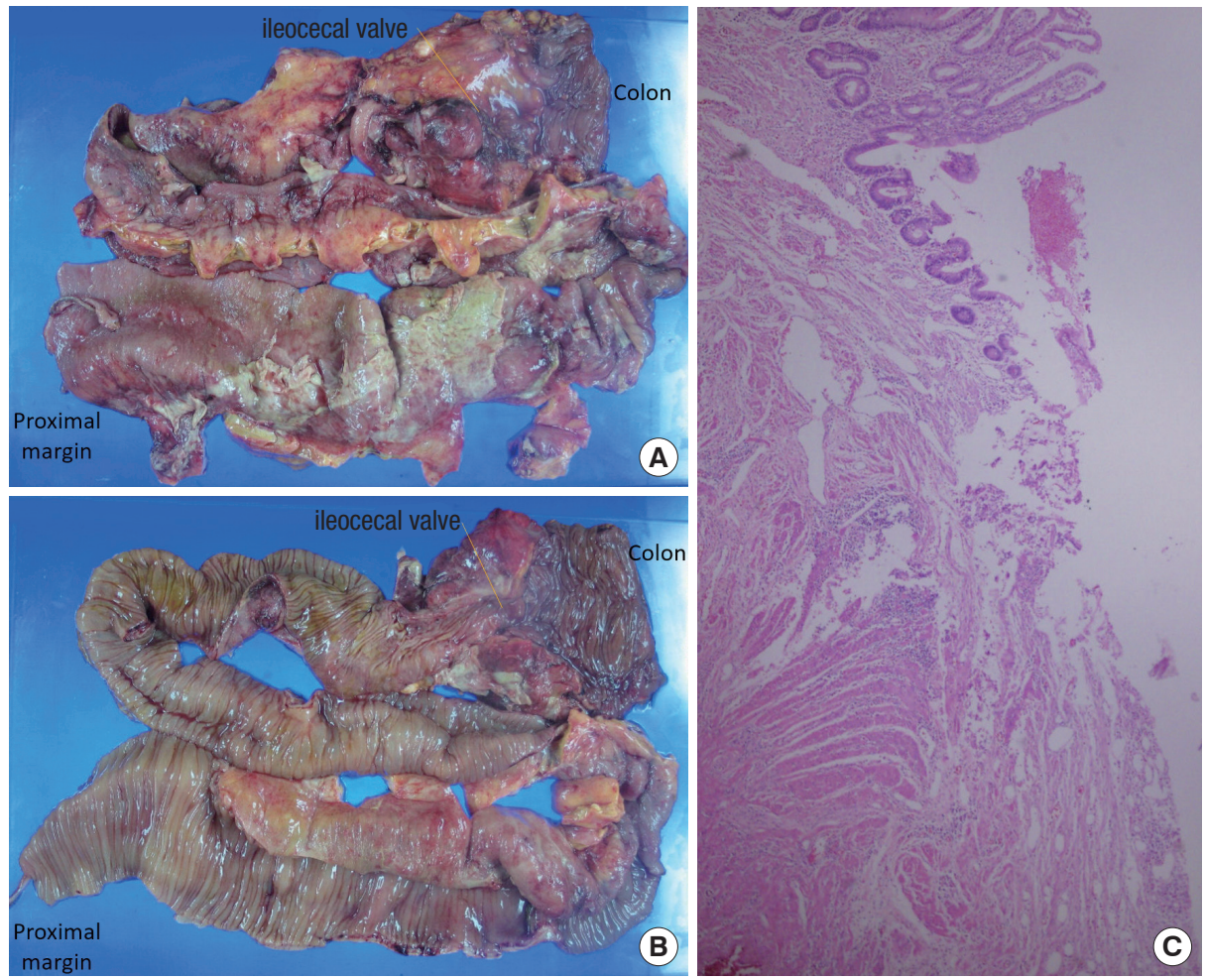

Fig. 5. (A) Intraoperative photograph showing suppurative inflammation on serosal surface of small bowel. (B) Luminal surface of the specimen. (C) Section of the resected bowel showing a perforated ulcer and acute, transmural, suppurative inflammation $(\mathrm{H} \& \mathrm{E}, \times 40)$.

ticosteroid therapy, anastomotic leaks, advanced age, and primary closure of the perforation $[3,4]$. Considering the high mortality associated with intestinal TB, an operative resection of the affected portion of the intestine, followed by anastomosis, is preferred to primary closure [6]. Intestinal TB occurs mainly in the ileocecal region, with perforation commonly involving the ileal mucosa. Perforation can occur at single or multiple sites and may be associated with the formation of intestinal strictures or ulceration. As most perforations are stricture-related, small-intestinal perforations are more common than large-intestinal perforations. Perforation may occur before or after starting antitubercular therapy [3]. Intestinal perforation occurring during or after completion of antitubercular therapy can be a paradoxical reaction to treatment.

Paradoxical reactions have been observed in patients with TB affecting the nervous system, respiratory system, skin/soft tissue, lymph nodes, and the abdomen, in decreasing order of frequency. According to one report, approximately $75 \%$ of patients experience worsening of their primary lesions, and approximately $25 \%$ develop new lesions at other sites [5]. Risk factors for paradoxical reactions include extrapulmonary tuberculous lesions, a relatively low basal lymphocyte level in peripheral blood, and a sudden rise in the lymphocyte count during treatment [7].

The pathophysiological mechanism of paradoxical reaction to
TB treatment is not fully understood. Increased exposure to mycobacterial antigens released from the bacilli, killed due to effective antitubercular therapy, strengthens delayed hypersensitivity of the host. Differential diagnoses of paradoxical reactions include diagnostic errors, inadequate response due to drug resistance, and poor adherence to therapy. Therefore, it is important to culture the affected tissue specimen at the time of the initial diagnosis to confirm the diagnosis and to determine the baseline level of drug resistance [2].

It is difficult to distinguish paradoxical reactions from treatment failures. When paradoxical reaction is suspected, continuing treatment is necessary. Surgeons may help determine appropriate surgical procedures for conditions such as intestinal TB, when paradoxical reactions require surgical treatment.

Our patient had 2 paradoxical reactions. The first was a paradoxical respiratory reaction. The initial chest X-ray had shown increased opacity of the right upper lobe, despite the absence of respiratory symptoms, such as cough and expectoration. He developed respiratory symptoms 4 weeks after starting antitubercular therapy, with findings of active TB on the chest CT. Some patients develop negative sputum AFB staining and culture results after completing 1 month of antitubercular therapy. Our patient experienced relief of his respiratory symptoms with concomitant improvement in his radiological findings after completing 6 


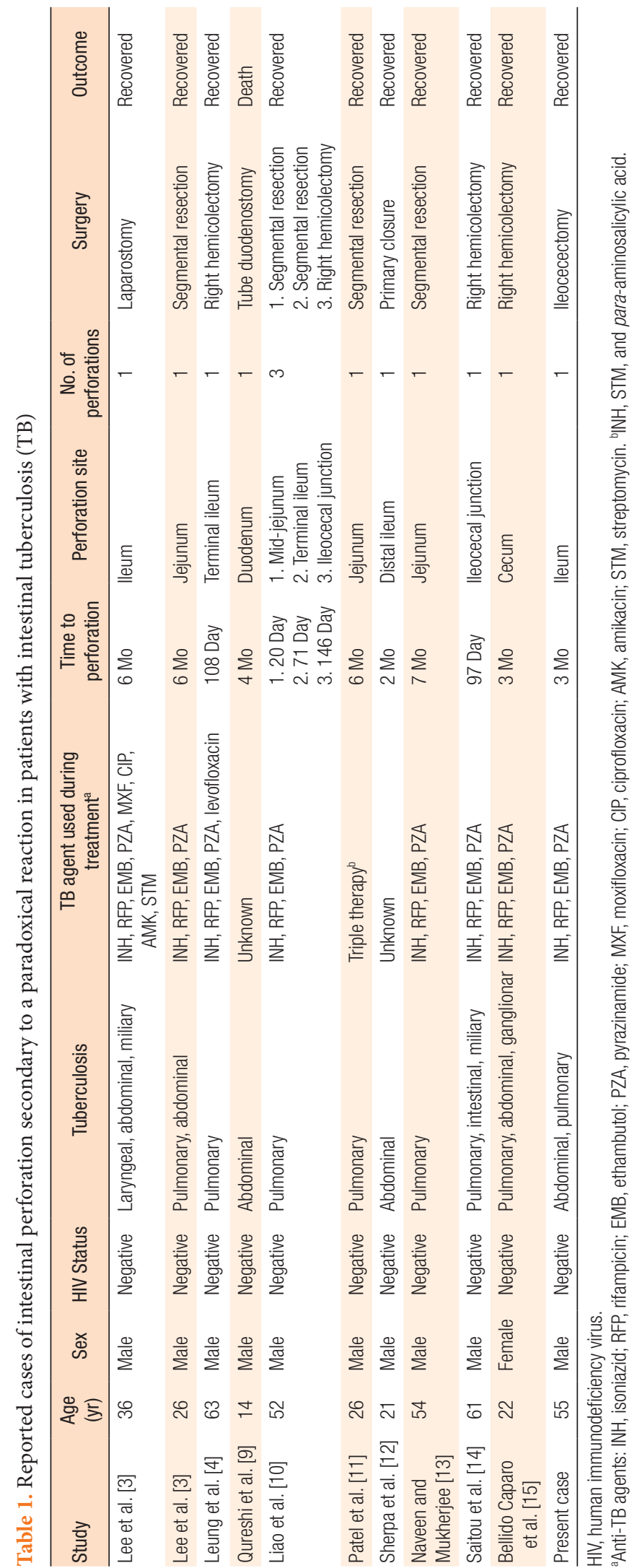

months of treatment. The second paradoxical reaction was the intestinal reaction. The patient's abdominal pain decreased after 3 months of treatment, and colonoscopy revealed an improvement in ulcerative lesions. However, the patient subsequently suffered a small bowel perforation, which required emergency surgical intervention. It was unclear whether the lesions were preexisting or new lesions because there was no test for small intestine lesions before treatment began. It is thought to be a clinical deterioration of the lesion that was already present. Since the patient had already been treated for 3 months, the TB lesions were not clearly visible in the postoperative biopsy. Blumberg et al. [8] recommended that a diagnosis of paradoxical reaction should be considered if symptomatic deterioration occurs within 3 months of starting antituberculous drugs, while that occurring 4 months or more after may be attributable to treatment failure or multidrugresistance. Our patient experienced worsening of his clinical symptoms within 3 months, increasing the likelihood of it being a paradoxical reaction.

Table 1 summarizes case reports of intestinal perforation occurring as a paradoxical reaction [3, 4, 9-15]. The mean age of affected patients was 39 years, and 10 out of 11 were males. The primary sites affected were mostly the lung and the abdomen. It took an average of 4.1 months from the initiation of antitubercular drugs to the occurrence of perforation. Therefore, it is important to carefully monitor patients with intestinal TB for intestinal perforations undergoing treatment for intestinal TB. Most patients underwent segmental resection and right hemicolectomy. The antitubercular drugs were continued after surgery, and most patients showed good postoperative recovery except for one patient with duodenal perforation [9].

We diagnosed our patient with ileocecal TB and started antitubercular therapy. However, as the initial evaluation of his small bowel lesion was inadequate, and so we were unable to predict the risk of occurrence of a paradoxical reaction. Therefore, in patients with suspected intestinal TB, evaluation of small bowel lesions with abdominal CT, in addition to colonoscopy, may be helpful in early diagnosis and enable prompt surgical treatment of complications such as small bowel perforation.

In conclusion, this case report describes a patient who experienced a small bowel perforation as a paradoxical reaction to the treatment for intestinal TB. We recommend a thorough baseline evaluation of the small bowel be conducted to assist clinicians with the prevention, diagnosis, and management of small bowel perforations occurring as a paradoxical reaction to antitubercular therapy.

\section{CONFLICT OF INTEREST}

No potential conflict of interest relevant to this article was reported. 


\section{REFERENCES}

1. World Health Organization. Global tuberculosis report 2019 [Internet]. Geneva: World Health Organization; 2019 [cited 2020 Aug 27]. Available from: https://www.who.int/tb/publications/ global_report/en/.

2. Golden MP, Vikram HR. Extrapulmonary tuberculosis: an overview. Am Fam Physician 2005;72:1761-8.

3. Lee MJ, Cresswell FV, John L, Davidson RN. Diagnosis and treatment strategies of tuberculous intestinal perforations: a case series. Eur J Gastroenterol Hepatol 2012;24:594-9.

4. Leung VK, Chu W, Lee VH, Chau TN, Law ST, Lam SH. Tuberculosis intestinal perforation during anti-tuberculosis treatment. Hong Kong Med J 2006;12:313-5.

5. Cheng VC, Ho PL, Lee RA, Chan KS, Chan KK, Woo PC, et al. Clinical spectrum of paradoxical deterioration during antituberculosis therapy in non-HIV-infected patients. Eur J Clin Microbiol Infect Dis 2002;21:803-9.

6. Ara C, Sogutlu G, Yildiz R, Kocak O, Isik B, Yilmaz S, et al. Spontaneous small bowel perforations due to intestinal tuberculosis should not be repaired by simple closure. J Gastrointest Surg 2005; 9:514-7.

7. Cheng VC, Yam WC, Woo PC, Lau SK, Hung IF, Wong SP, et al. Risk factors for development of paradoxical response during antituberculosis therapy in HIV-negative patients. Eur J Clin Microbiol Infect Dis 2003;22:597-602.
8. Blumberg HM, Burman WJ, Chaisson RE, Daley CL, Etkind SC, Friedman LN, et al. American Thoracic Society/Centers for Disease Control and Prevention/Infectious Diseases Society of America: treatment of tuberculosis. Am J Respir Crit Care Med 2003; 167:603-62.

9. Qureshi AU, Khan HM, Gondal KM. Duodenal perforation in a 14-year-old boy with abdominal tuberculosis despite being on antituberculous treatment. Indian J Surg 2010;72:69-70.

10. Liao CZ. Recurrent small intestinal perforations during anti-tuberculous treatment. Formos J Surg 2012;45:161-3.

11. Patel S, Al-Nowfal A, Gould ST. A rare cause of faecal peritonitis: jejunal perforation in a patient undergoing treatment for pulmonary tuberculosis. J Surg Case Rep 2012;2012:rjs014.

12. Sherpa MT, Shrestha R, Limbu PM. Multiple intestinal strictures with perforation in a patient under antitubercular treatment for abdominal tuberculosis. J Nepal Health Res Counc 2013;11:86-8.

13. Naveen N, Mukherjee A. A rare case of perforation peritonitis with jejunal stricture in a patient recently treated for pulmonary tuberculosis. J Clin Diagn Res 2014;8:ND07-8.

14. Saitou M, Suzuki T, Niitsuma K. Intestinal perforation due to paradoxical reaction during treatment for miliary tuberculosis. Respirol Case Rep 2016;4:e00196.

15. Bellido Caparo A, Pampa-Espinoza L, Espinoza-Rios J. Cecal perforation secondary to a paradoxical reaction in a patient with disseminated tuberculosis: case report and review of the literature. Gastroenterol Hepatol Open Access 2018;9:272-5. 